\title{
Current Perspectives on Aerobic Exercise in People with Parkinson's Disease
}

\author{
Sabine Schootemeijer ${ }^{1}$ (D) Nicolien M. van der Kolk ${ }^{1} \cdot$ Bastiaan R. Bloem ${ }^{1} \cdot$ Nienke M. de Vries ${ }^{1}$
}

Published online: 17 August 2020

(C) The Author(s) 2020

\begin{abstract}
Parkinson's disease (PD) is a progressive neurological disorder characterized by motor and non-motor symptoms for which only symptomatic treatments exist. Exercise is a widely studied complementary treatment option. Aerobic exercise, defined as continuous movement of the body's large muscles in a rhythmic manner for a sustained period that increases caloric requirements and aims at maintaining or improving physical fitness, appears promising. We performed both a scoping review and a systematic review on the generic and disease-specific health benefits of aerobic exercise for people with PD. We support this by a metaanalysis on the effects on physical fitness $\left(\mathrm{VO}_{2} \mathrm{max}\right)$, motor symptoms (Movement Disorder Society Unified Parkinson's Disease Rating Scale (MDS-UPDRS) motor section), and health-related quality of life (39-item Parkinson's disease Questionnaire (PDQ39)). Aerobic exercise has generic health benefits for people with PD, including a reduced incidence of cardiovascular disease, a lower mortality, and an improved bone health. Additionally, there is level 1 evidence that aerobic exercise improves physical fitness $\left(\mathrm{VO}_{2} \mathrm{max}\right)$ and attenuates motor symptoms (MDS-UPDRS motor section) in the off-medication state, although the longterm effects (beyond 6 months) remain unclear. Dosing the exercise matters: improvements appear to be greater after training at higher intensities compared with moderate intensities. We found insufficient evidence for a beneficial effect of aerobic exercise on health-related quality of life (PDQ-39) and conflicting results regarding non-motor symptoms. Compliance to exercise regimes is challenging for PD patients but may be improved by adding exergaming elements to the training program. Aerobic exercise seems a safe intervention for people with PD, although care must be taken to avoid falls in at-risk individuals. Further studies are needed to establish the long term of aerobic exercise, including a focus on non-motor symptoms and health-related quality of life.
\end{abstract}

Key Words Exercise $\cdot$ Parkinson's disease $\cdot$ endurance training $\cdot$ locomotion $\cdot$ health-related quality of life $\cdot$ mobility limitation

\section{Introduction}

Parkinson's disease (PD) is a progressive neurological disorder characterized by both motor and non-motor symptoms. The well-known motor symptoms include bradykinesia, problems with gait and balance, tremor, and rigidity. The non-motor symptoms are diverse and can, among others, include fatigue, cognitive dysfunction, depression, and apathy. The prevalence of PD

Bastiaan R. Bloem

bas.bloem@radboudumc.nl

1 Donders Institute for Brain, Cognition and Behavior, Department of Neurology, Center of Expertise for Parkinson \& Movement Disorders, Radboud University Medical Center, PO Box 9101, 6500 HB Nijmegen, Netherlands is vastly increasing, and by 2040, almost 13 million people worldwide will be affected by PD [1]. Unfortunately, there is neither a cure nor a disease-modifying treatment for PD. Current treatment mainly consists of dopaminergic replacement strategies (medication or deep brain surgery) aimed at alleviating PD symptoms. However, the effectiveness of the pharmacological treatment tends to wear off over time, resulting in disabling motor fluctuations and dyskinesias [2]. Moreover, not all patients are eligible for advanced therapies, which themselves are not without risks or side effects [3]. Taken together, there is a pressing need for alternative therapeutic strategies that reduce disability and improve health-related quality of life. Exercise (see Box 1 for a definition) is increasingly being recognized as an effective and highly promising non-pharmacological intervention to improve physical function. High-quality evidence on the diseasespecific effects of exercise is relatively scarce [4], although several well-designed studies have been published in recent years. 
Box 1 Exercise terminology according to the American College of Sports Medicine

\begin{tabular}{ll}
\hline Term & Definition \\
\hline Physical activity & $\begin{array}{c}\text { Any bodily movement produced by the contraction } \\
\text { of skeletal muscles that results in a substantial } \\
\text { increase in caloric requirements over resting } \\
\text { energy expenditure }\end{array}$ \\
Exercise & $\begin{array}{c}\text { A type of physical activity that consists of planned, } \\
\text { structured, and repetitive body movements that are } \\
\text { performed to improve or maintain 1 or more } \\
\text { components of physical fitness }\end{array}$ \\
Aerobic exercise & $\begin{array}{c}\text { A subcategory of exercise that involves continuous } \\
\text { movements of the body's large muscles in a } \\
\text { rhythmic manner for sustained periods }\end{array}$ \\
\hline
\end{tabular}

In this review, we focus on aerobic exercise, a type of exercise that involves continuous movements of the body's large muscles in a rhythmic manner for sustained periods, increases heart rate and caloric requirements, and is performed to maintain or improve physical fitness [5]. Different lines of evidence (animal models of PD [6-9], small human studies $[10,11]$, longitudinal cohort studies [12-16]) underlined the therapeutic potential of aerobic exercise in PD and identified aerobic exercise as a promising form of exercise to study in people with PD. A previous review of clinical trials investigating aerobic exercise in PD concluded that aerobic exercise improves physical fitness in PD, but evidence on diseasespecific effects was lacking [17]. During the last years, the number of high-quality randomized clinical trials investigating the effect of aerobic exercise on PD symptoms (both motor and non-motor) has increased. In this comprehensive review, we provide an update of the current literature regarding the effect of aerobic exercise in people with PD (Fig. 1). In addition to the disease-specific effects of aerobic exercise, we also review the generic health benefits and issues related to implementation (safety and adherence).

\section{Methods}

We review the effects of aerobic exercise on multiple domains in people with $\mathrm{PD}$, including generic health benefits, diseasespecific effects (on both motor and non-motor functioning), impact on health-related quality of life, and issues related to implementation. Because not all domains are studied in clinical trials, we combine narrative and systematic review techniques. The generic health benefits (cardiovascular health and risk factors, bone health, and fractures), prevention of $\mathrm{PD}$, and implementation issues (safety and adherence) are described in a narrative way. The effects of aerobic exercise on physical fitness, on disease-specific motor and non-motor functioning, and on health-related quality of life are addressed in a systematic review. For the latter, we built upon a review [17] that included aerobic exercise trials published between 1990 and August 1, 2014. We systematically searched for randomized clinical trials from August 1, 2014 (i.e., the date of the search of the previous review [17] until February 19, 2020. The search strategy is available in Supplementary Box 1. In addition, we performed citation tracking through the included randomized clinical trials. We used similar inclusion criteria as before [17]: 1) target population consisting of people with PD, 2 ) aerobic exercise group exercising with at least an intensity of $60 \%$ of maximal heart rate (HRmax) [18], 3) intervention lasting at least 4 weeks, and 4 ) an aerobic exercise group was compared with a control or other non-aerobic exercise groups. We excluded one study [19] that was included in the earlier

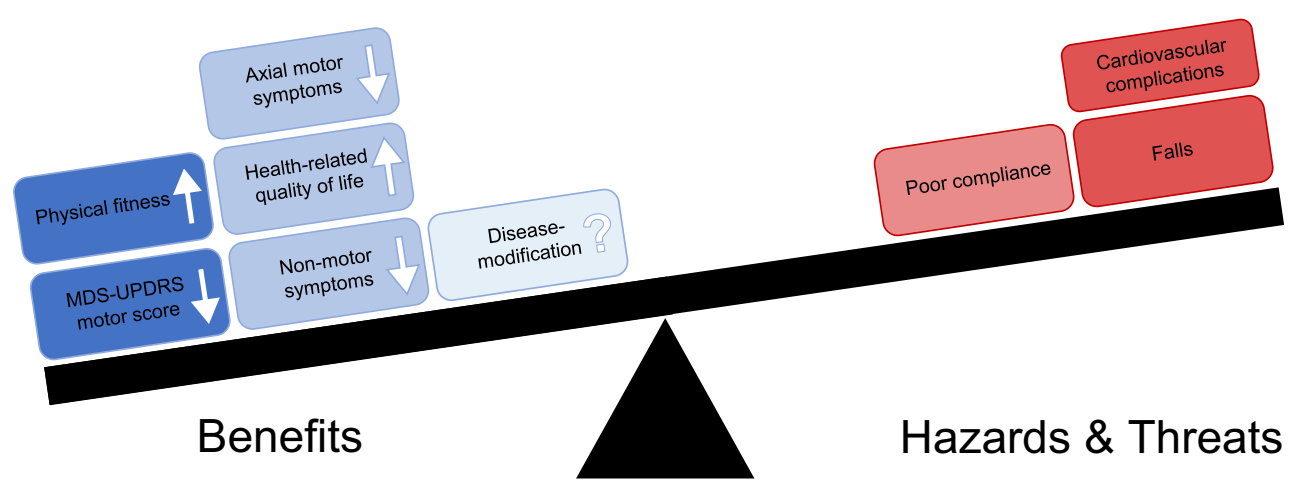

\section{Advice to exercise}

Fig. 1 Benefits, potential hazards, and threats of aerobic exercise. The seesaw is tilting towards the left, indicating that the benefits of exercise outweigh the potential hazards and threats of aerobic exercise for people with PD. Darker blue color represents more evidence for benefit. The red color represents a potential hazard of exercise itself, while the lighter red color represents a threat to participating in exercise. There is a theoretical concern that elderly and sedentary persons who start a vigorous exercise program may experience cardiovascular complications, but this risk appeared to be very low in published exercise trials involving sedentary persons with $\mathrm{PD}$. White arrows depict direction of change with aerobic exercise. MDS-UPDRS = Movement Disorders Society Unified Parkinson's Disease Rating Scale. 
review [17] because of a lack of control or other non-aerobic exercise groups.

To further support the evidence, we conducted a metaanalysis for a few selected outcomes: physical fitness (maximal oxygen consumption, $\mathrm{VO}_{2} \max$ ), motor function (Movement Disorder Society Unified Parkinson's Disease Rating Scale (MDS-UPDRS) motor section) in the on- and off-medication state and health-related quality of life (39-item Parkinson's Disease Questionnaire (PDQ-39)). We used the post-intervention scores of the exercise group and control group (between-group difference) to calculate a pooled mean difference (MD) with $95 \%$ confidence intervals (CIs) of the $\mathrm{VO}_{2}$ max and PDQ-39. For the (MDS-)UPDRS motor section, we calculated the standardized mean difference (SMD) scores, because we had to combine two different outcome measures (the UPDRS motor section and the MDS-UPDRS motor section). Post-intervention scores were used, because they were presented in most of the studies and change scores were often not available. (Standardized) mean differences were pooled with the inverse variance method. Homogeneity of variances was evaluated with the $I^{2}$ test [20], and if heterogeneity was absent $\left(I^{2}, 0-25 \%\right)$, we applied a fixed effects model. Heterogeneity was present for the (MDS-)UPDRS motor section; for this reason, we applied a random effects model. We examined funnel plots to assess the presence of publication bias, but the risk of publication bias was low [21].

\section{Quality of Included Studies}

The quality of the included randomized clinical trials was assessed by two reviewers (SS and NMdV) independently with the risk of bias tool [22]. Quality assessments were
Fig. 2 Summary of the risk of bias of randomized clinical trials included in the systematic review.
Study

Altmann 2013

Bergen 2002

Bridgewater 1996

Carvalho 2015

Cugusi 2015

Fisher 2008

Kurtais 2008

Marusiak 2019

Sacheli 2019

Sage 2009

Schenkman 2012

Schenkman 2018

Shulman 2013

Silveira 2018

Tollar 2018

Van der Kolk 2018

Van der Kolk 2019

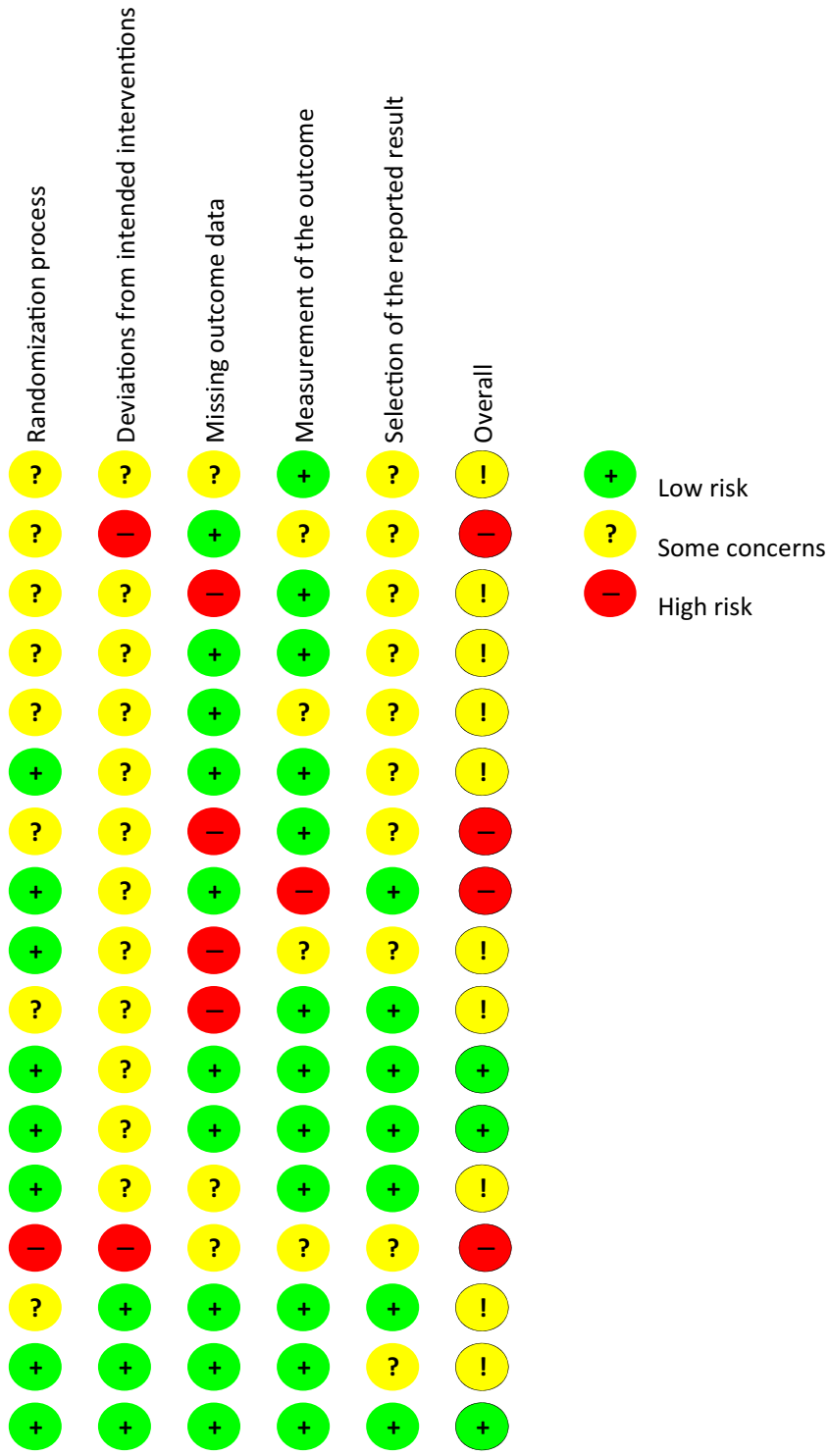


compared and discussed when necessary. In total, three studies were rated with the low risk of bias [4, 23, 24], 10 studies with some concerns about the risk of bias [11, 25-33], and four studies with the high risk of bias [34-37] (Fig. 2). The risk of bias was mainly based on 1) unclear randomization procedure and concealment; 2) lack of blinding of participants, therapists delivering the intervention and assessors; 3 ) unclear or incomplete reporting of dropouts; and 4) lack of a published prespecified statistical analysis plan. Blinding participants and therapists is often challenging when delivering an aerobic exercise intervention. Very few trials included a non-exercise group that received similar social interaction to blind the participants. In general, the newer studies were of higher quality than the older studies.

\section{Results}

Table 1 provides an overview of the methods and major findings of the 17 randomized clinical trials included in this systematic review. Figure 1 provides an overview of the overall evidence for the effect of aerobic exercise. We suggest that the benefits of aerobic exercise outweigh the potential hazards and risks.

\section{Generic Health Benefits}

\section{Cardiovascular Health}

Aerobic exercise has generic health benefits for almost everyone, including people with a chronic disease like PD [5]. In older adults, regular physical activity is associated with a lower risk of cardiovascular disease and cardiovascular mortality [5]. There is a clear dose-effect relation between aerobic exercise and cardiovascular health and mortality: the more time spent on aerobic exercise at moderate intensity, the lower the cardiovascular mortality [38]. For exercise at vigorous intensity, the same dose-effect relationship holds true, except beyond $11 \mathrm{MET} \mathrm{h} /$ week, which does not lead to a further reduction in cardiovascular mortality [38].

Aerobic exercise also impacts on the metabolic system. Metabolic syndrome is characterized by the occurrence of several risk factors for cardiovascular disease (insulin resistance, hyperlipidemia, insulin resistance, hypertension) [39]. Exercise can prevent metabolic syndrome and can also result in reversal of muscle insulin resistance and reduction of postprandial lipogenesis [39]. These findings are also relevant for PD. Specifically, in one cohort study $(N=1.022)$, metabolic syndrome was associated with a faster deterioration in motor symptoms of PD [40]. Another cohort study found an association with cognitive decline in PD patients [41]. However, whether exercise can revert the metabolic syndrome (and its consequences) in PD patients remains to be studied.
Another interesting area relates to cerebral small vessel disease, including lacunar infarcts and white matter hyperintensities, which commonly occur as comorbidity in PD patients [42, 43]. Importantly, the concurrent presence of small vessel disease is associated with a poorer course of PD, with worsening of gait problems, cognitive decline, and depression $[42,44]$. A lack of physical activity among PD patients may further aggravate this risk of developing comorbid small vessel disease. Theoretically, exercise could mitigate this risk, certainly when extrapolating the observed protective effects in the general population. However, a prophylactic effect of aerobic exercise on cerebrovascular disease has thus far not been studied in persons with PD.

\section{Bone Health and Fractures}

Regular physical activity is associated with improved bone health in older adults [5]. People with PD are generally less physically active than their healthy counterparts, which may contribute to the development of osteoporosis in PD [45]. Indeed, osteoporosis is very common in people with PD [46], who have a lower bone mineral density and reduced bone strength compared with healthy people [45, 47]. Apart from inactivity, other contributing factors include vitamin D deficiency, reduced muscle strength, poor mobility, and hyperhomocysteinemia (i.e., catabolism of homocysteine, a status associated with risk of fractures and a low bone mineral density; this status is possibly aggravated by levodopa use) [45]. Along with their gait deficits and postural instability (leading to falls), osteoporosis places people with PD at risk of sustaining fractures [46]. Moreover, the combination of $\mathrm{PD}$ and osteoporosis has been associated with pain, sleep problems, depression, and anxiety [48]. These consequences might be countered by weight-bearing aerobic exercise (brisk walking, running), which stresses the bones and thereby promotes bone growth and bone strength in healthy older adults [5]. The effect of aerobic exercise on bone health and osteoporosis in PD remains to be shown, but simple interventions such as brisk walking seem promising.

\section{Preventing Parkinson's Disease}

Epidemiological studies suggest that in the general population, people who are physically more active may be at lower risk of developing PD [13-16, 49-52]. Interestingly, premorbid physical activity also seems to influence disease symptom onset [52, 53]. For example, the chance of developing prodromal features of PD (constipation, bodily pain, depression, and excessive daytime sleepiness) is negatively associated with physical activity [54], as is motor symptom onset [53]. It is unclear whether physical activity and aerobic exercise can actually prevent or delay the development of PD itself (i.e., by slowing the underlying neurodegenerative 


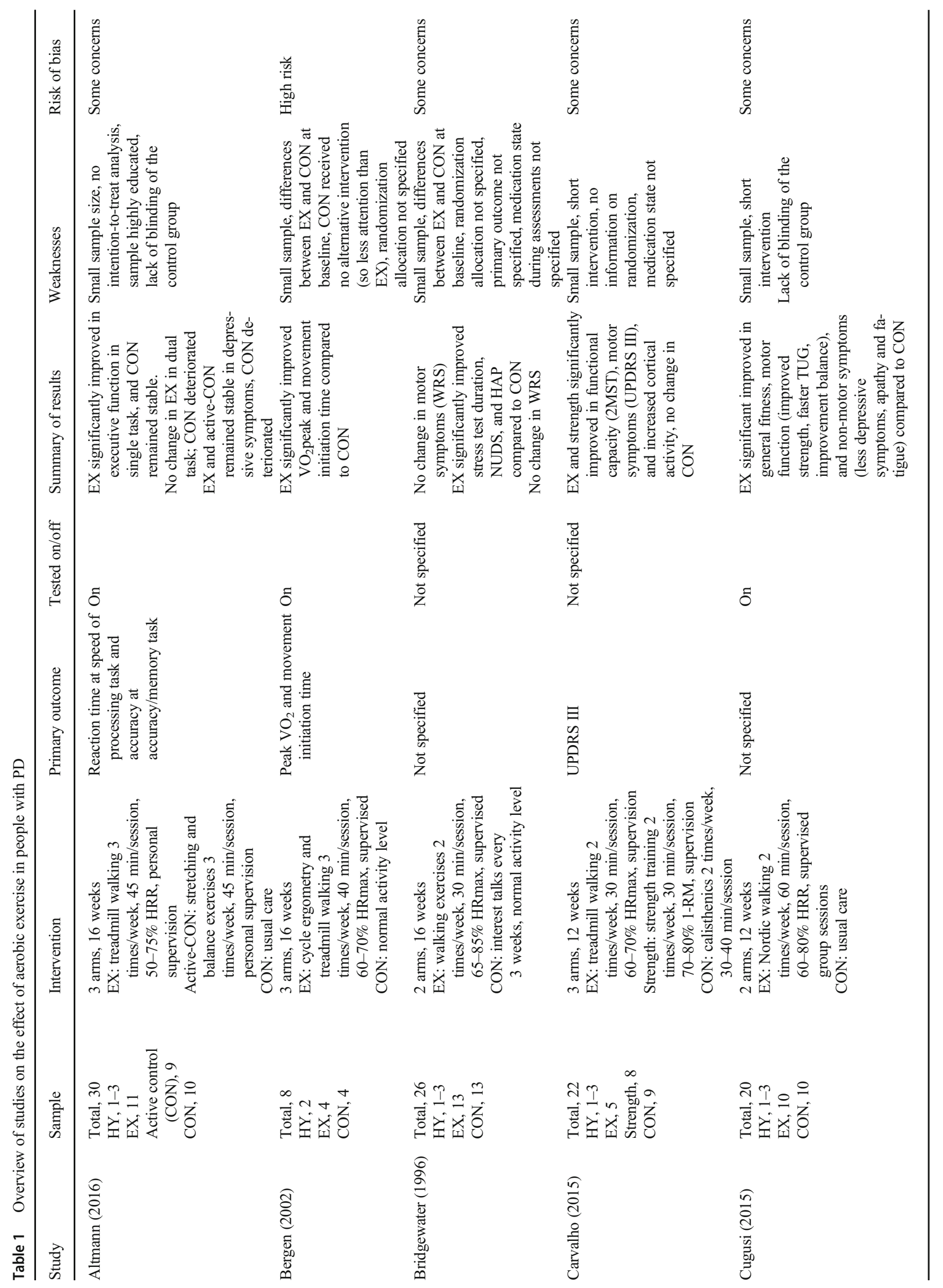




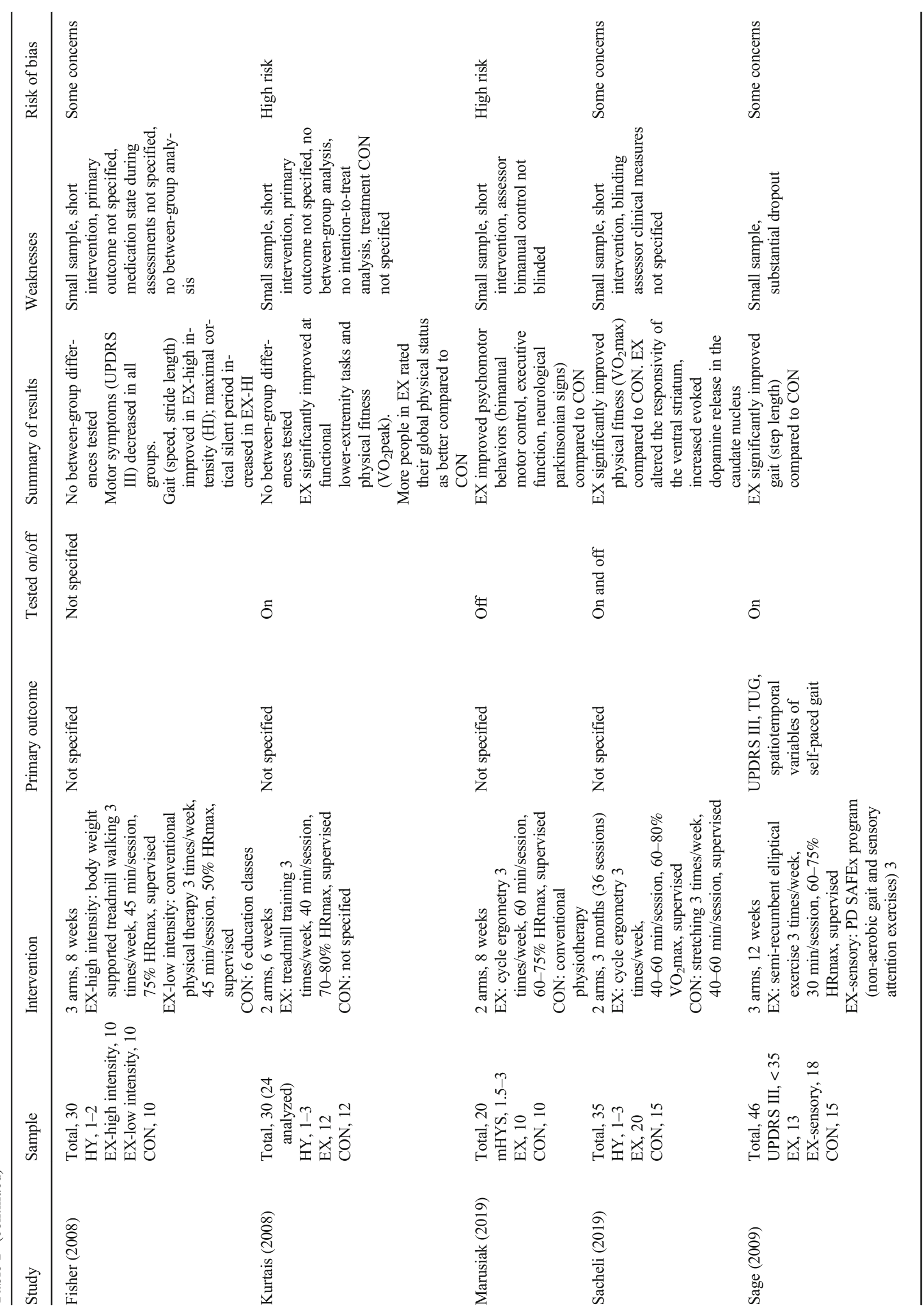




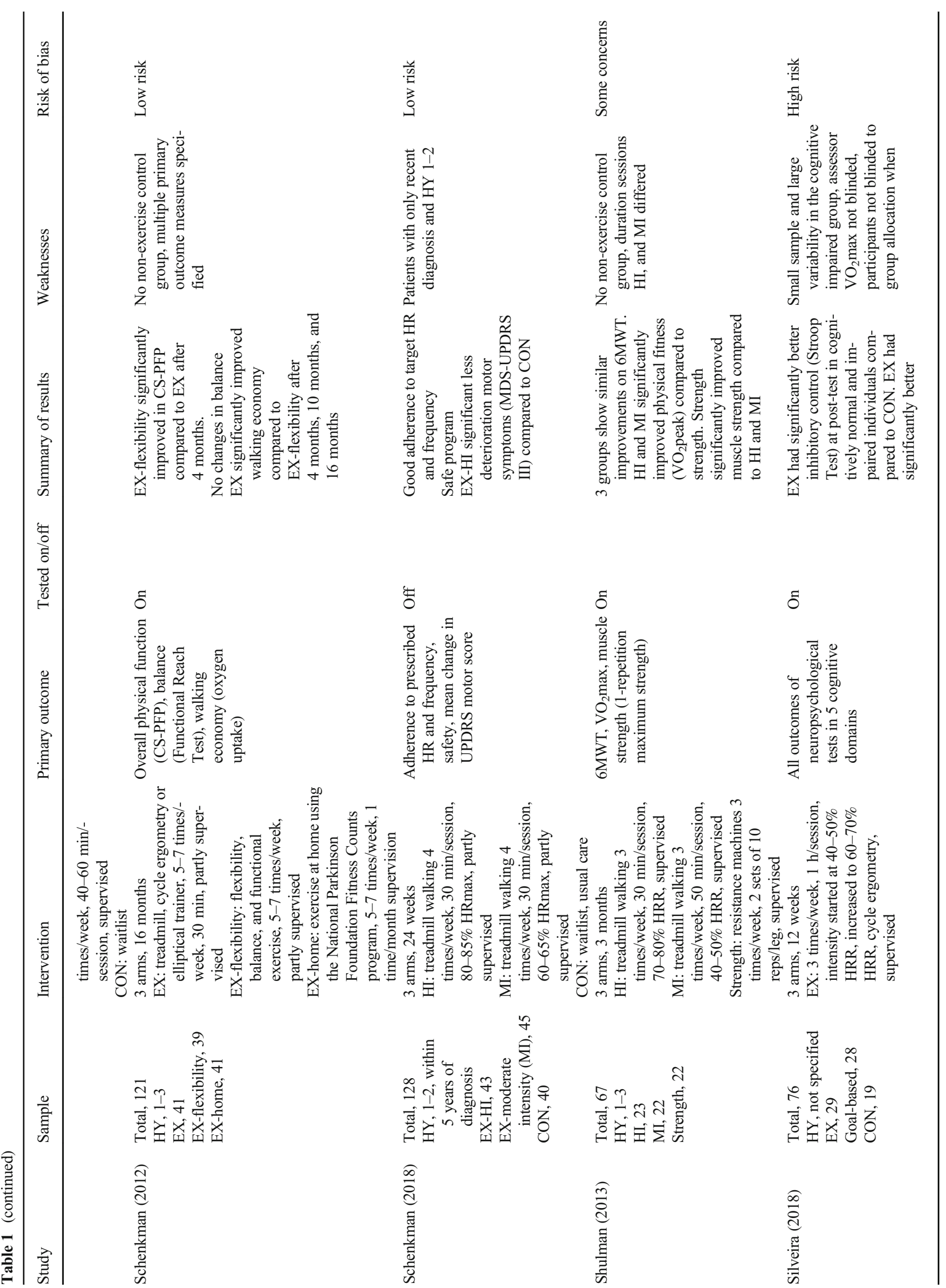




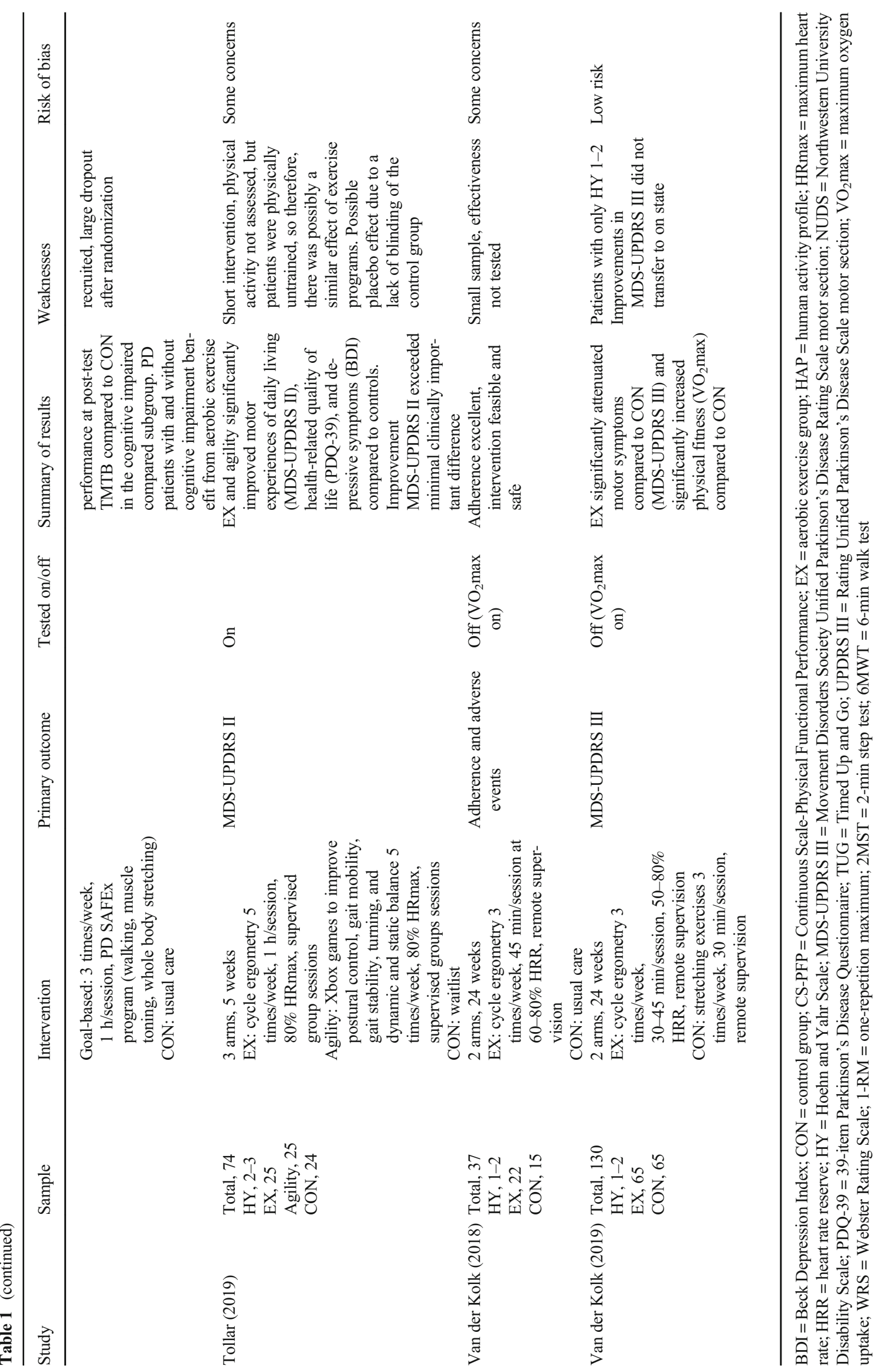


process itself) or whether this induces compensatory effects in other brain areas that delay the manifestation of PD symptoms. Further research into the underlying mechanisms of the potential neuroprotective effect of physical activity and exercise is needed [55] and is also elaborated upon in the discussion of this review.

\section{Symptomatic Effects in Manifest Parkinson's Disease}

\section{Physical Fitness}

Aerobic exercise improves physical fitness in people with PD (Fig. 3). Ten randomized controlled trials investigated the effect of aerobic exercise on physical fitness, as assessed with a (sub)maximal graded exercise test $\left(\mathrm{VO}_{2} \max \right)$ [4, 11, 23, 24, 29, 31, 33-35, 37]. All studies showed a beneficial effect of aerobic exercise on physical fitness, although two studies only reported within-group effects $[11,35]$. Different exercise types were studied but generally involved (treadmill) walking or cycling on a stationary bicycle. The studies also differed in length, varying from 6 to 24 weeks, and used different training frequencies (3-7 times/week) and intensities (60-85\% HRmax, 60-80\% $\mathrm{VO}_{2} \max$, or $50-80 \%$ heart rate reserve). Two studies investigated the difference in effectiveness of low/moderate-intensity versus high-intensity aerobic exercise training [23, 31]. One of these studies showed similar improvements in physical fitness. However, in this study, the duration of the training sessions also differed, which makes it impossible to conclude whether duration or intensity of the exercise contributed to these effects [31]. The other high-quality trial showed that high-intensity exercise for 6 months was more effective in improving physical fitness than moderate-intensity exercise [23]. Overall, these studies provide level 1 evidence for a beneficial effect of aerobic exercise on physical fitness in PD. This positive effect is also supported by our meta-analysis (Box 2).

\section{Box 2 Results of meta-analyses}

Physical fitness

The positive effect of aerobic exercise on physical fitness is supported by the meta-analysis: the aerobic exercise group showed a higher physical fitness during a maximal graded exercise test $\left(\mathrm{VO}_{2} \max \right)$ at the post-assessment compared with a non-exercise or resistance exercise control group (mean difference (MD) $[95 \% \mathrm{CI}], 2.25[1.90 ; 2.60]$; $Z=12.66 ; p<0.05$ ) (Fig. 3)

Motor symptoms in PD

The meta-analysis on (MDS)-UPDRS shows that patients with PD who have performed aerobic exercise experienced less motor symptoms at the post-assessment compared with the a non-aerobic control group when assessed in the off state [4, 23, 29, 33] (SMD [95\% CI], $-0.42[-0.77$; -0.08 ]; $Z=2.39 ; p=0.02$ ) (Fig. 4B), but not when assessed in the on state $[24,25,28,30,31]$ (SMD [95\% CI], $-0.21[-0.72 ; 0.30] ; Z=0.81$, non-significant) (Fig. 4A). Two studies [11, 27] were excluded from the analysis of the (MDS-UPDRS) because medication state was not reported

Health-related quality of life People in the aerobic exercise group did not experience a better health-related quality of life at the post-assessment compared with those in the non-aerobic control group (MD [95\% CI], -0.31 $[-1.05 ; 0.43] ; Z=0.82$, non-significant) (Fig. 5)

\section{Physical Functioning}

Motor Symptoms of PD Aerobic exercise likely has a beneficial effect on PD motor symptoms (Fig. 4). Eleven randomized controlled trials investigated the effect of aerobic exercise on motor symptoms, as assessed with the (MDS-)UPDRS motor section $[4,11,23-25,27-31,33]$. This was the primary

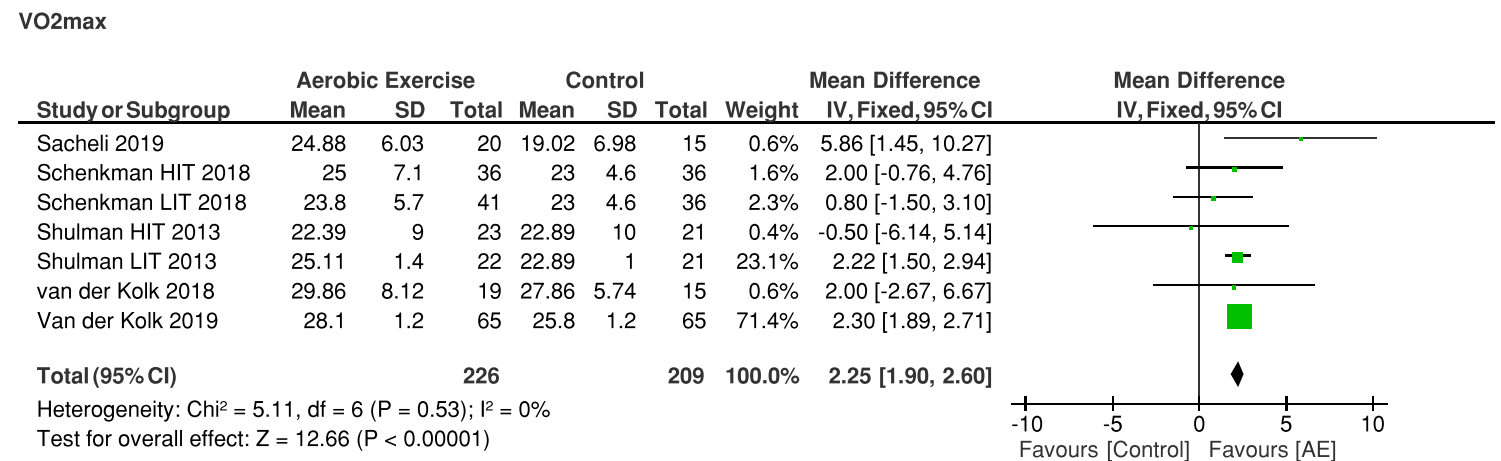

Fig. 3 Meta-analysis on the effect of aerobic exercise on physical fitness in $\mathrm{PD}$ comparing post-intervention $\mathrm{VO}_{2}$ max between the aerobic exercise group and the control group. AE = aerobic exercise group; HIT = high-intensity group; LIT = low-intensity group. 
a

(MDS-)UPDRS-III on

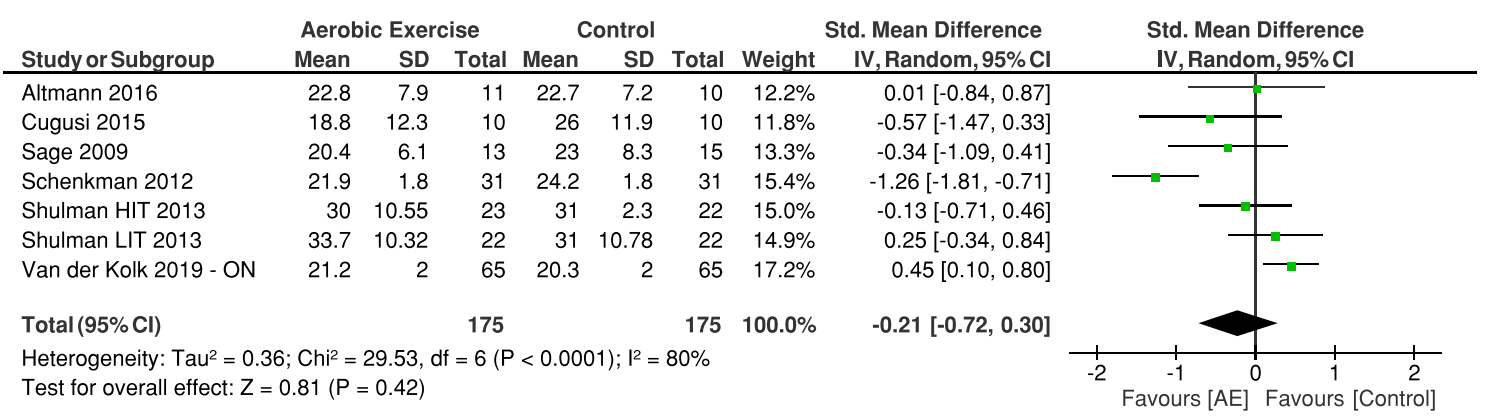

b

(MDS-)UPDRS-III off

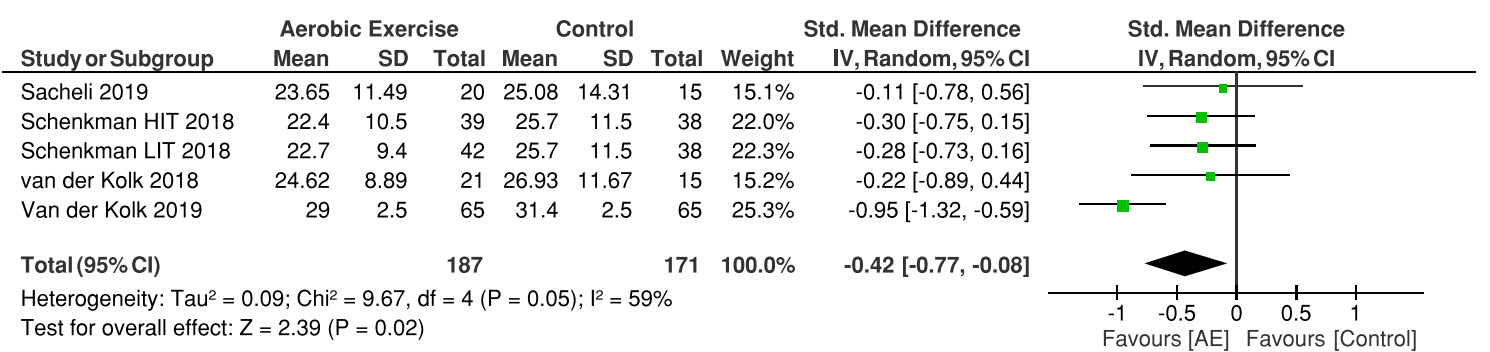

Fig. 4 Meta-analysis on the effect of aerobic exercise on motor function in PD comparing post-intervention (MDS-)UPDRS motor section between the aerobic exercise group and the control group in the medication

outcome in four studies $[4,23,27,30]$. Three studies showed that motor symptoms either improved (when measured in the on-medication state) [28] or attenuated (when measured in the off-medication state) $[4,23]$. This was not confirmed in the eight other studies [11, 24, 25, 27, 29-31, 33]. However, these latter studies were generally of lower methodological quality. Moreover, our meta-analysis shows an overall positive effect of aerobic exercise on motor symptoms in the off but not in the on state (Box 2). The long-term effects (beyond 6 months) remain unclear.

Other Aspects of Physical Functioning The preceding paragraph clarifies that exercise has a beneficial effect on a composite score for multiple motor domains. Other research examined whether exercise might also benefit specific isolated motor features such as gait, balance, falls, or functional mobility, but there is insufficient evidence for a beneficial effect here. Specifically, 12 randomized controlled trials investigated the effects of aerobic exercise on these motor symptoms, with conflicting results $[4,11,23,24,27-33,35]$. Two studies showed that aerobic exercise improved balance more than usual care $[28,32]$. However, the improvement did not transfer to other balance tests [32] and this finding was not confirmed by other studies $[4,24,27,33,35]$. Because of the specificity of training, we expected that gait-based interventions, for example using a treadmill, would have more positive on $(\mathbf{a})$ and off $(\mathbf{b})$ state. $\mathrm{AE}=$ aerobic exercise group; $\mathrm{HIT}=$ high-intensity group; LIT $=$ low-intensity group.

effects on functional mobility than non-gait-based interventions (i.e., cycling). However, this could not be confirmed by the available data, which showed that both types of interventions had no positive effect on physical functioning.

\section{Non-motor Symptoms}

Mood Disorders and Apathy Nine studies evaluated the impact of aerobic exercise on mood disorders [4, 25, 26, 28, 29, $31-33,36]$, and three studies also included apathy as outcome $[25,28,29]$. Two studies showed a positive effect of aerobic exercise on depressive symptoms $[28,32]$. This was, however, not confirmed by other trials $[4,25,26,29,31,33,36]$. For apathy, only one study showed a positive effect [28]. We conclude that there is insufficient evidence for a beneficial effect of aerobic exercise on mood disorders and apathy.

Cognition Six trials investigated the effect of aerobic exercise on cognition, with conflicting results $[4,25,29,33,36,37]$. Two studies showed that aerobic exercise improved executive function (Stroop Test) [25, 37]. Another study found a positive effect on mental flexibility (Trail Making Test B), specifically in persons with PD with cognitive impairments [36]. The other studies showed no change in cognitive functioning measured with different instruments $[4,29,33]$. We therefore 
conclude that there is insufficient evidence for a beneficial effect of aerobic exercise on cognition.

Sleep and Fatigue Sleep was included as a secondary outcome in three trials, none of which reported a positive effect of aerobic exercise [4, 25, 33]. Three trials also included fatigue as a secondary outcome $[4,28,31]$. One of these showed a positive effect [28] while the others showed no effect on fatigue. The study that did find a positive effect on fatigue also reported improvements in physical functioning, depressive symptoms, apathy, and overall burden of non-motor symptoms [28]. However, this study has several methodological shortcomings: the intervention was short (only 12 weeks), the sample was small $(N=20)$, and the control group was not blinded. Overall, there is currently insufficient evidence for a beneficial effect of aerobic exercise on sleep and fatigue.

Constipation Constipation is a common and vexing issue for people with PD. In daily practice, physical activity is commonly recommended as one possible intervention. Only one recent study took this to the test and included constipation as outcome, but the results showed no effect [4].

\section{Health-Related Quality of Life}

Aerobic exercise should ideally not just ameliorate isolated symptoms but also improve health-related quality of life (Fig. 5). A 2-year prospective cohort study in 3408 PD patients showed that health-related quality of life decreased less in people who exercised regularly $(\geq 2.5 \mathrm{~h} /$ week) as compared with non-exercisers [56]. However, these promising findings from observational data have not been confirmed in controlled clinical trials (which is also supported by our meta-analysis; Box 2). Only one study showed a beneficial effect of a highintensity (80\% HRmax) and high-frequency (5 times/week) aerobic exercise program [32]. The other five studies that evaluated health-related quality of life found no positive effects $[4,23,24,31,33]$.

\section{Implementation Issues}

\section{Adherence}

Although the evidence for the beneficial effects of exercise is accumulating, many patients with PD still do not adopt an active lifestyle in daily life. Many comply very well with prescribed exercise regimens within the context of clinical trials. Attrition across the aerobic exercise studies included in the systematic review ranged between $0 \%$ in small supervised trials $[11,28,34,36]$ and $25 \%$ in a program that was also personally supervised [25]. Adherence to the prescribed frequency, intensity, and duration of exercise is not often well reported. Attendance rates to on-site training sessions varied between 87 and $100 \%$ [25, 26, 28, 30, 32, 36], and adherence to exercise frequency in partly or unsupervised settings was still good (>75\% sessions attended) [4, 23, 33]. Although adherence is overall good in clinical trials, in daily life, many practical barriers must be overcome [57-64]. Barriers include a range of factors like low self-efficacy, generic health issues, poor access to the exercise location, mobility problems such as postural instability, and non-motor symptoms such as fatigue and depression [57-63, 65-71]. On the other hand, social support by family, friends, or a professional and education about the benefits of exercise can motivate PD patients to become more physically active $[57,59,60,62-69,72-74]$. At least 150 min of moderate-intensity exercise spread over multiple days is advised [5], but we find it helpful to instruct patients to exercise on a daily basis so it becomes part of their everyday routine. Healthcare professionals should be aware of these various barriers and facilitators, so these can be addressed and alleviated in a personalized approach, preferably using a sports coach. We have a positive experience with such coaching programs in two studies. The first was the ParkFit trial which deployed coaches who codesigned an individualized exercise program together with patients, after which patients signed a contract to commit themselves to the planned activities; compliance proved to be good [64]. The second was the Park-in-Shape trial in which coaches remotely supervised

PDQ-39

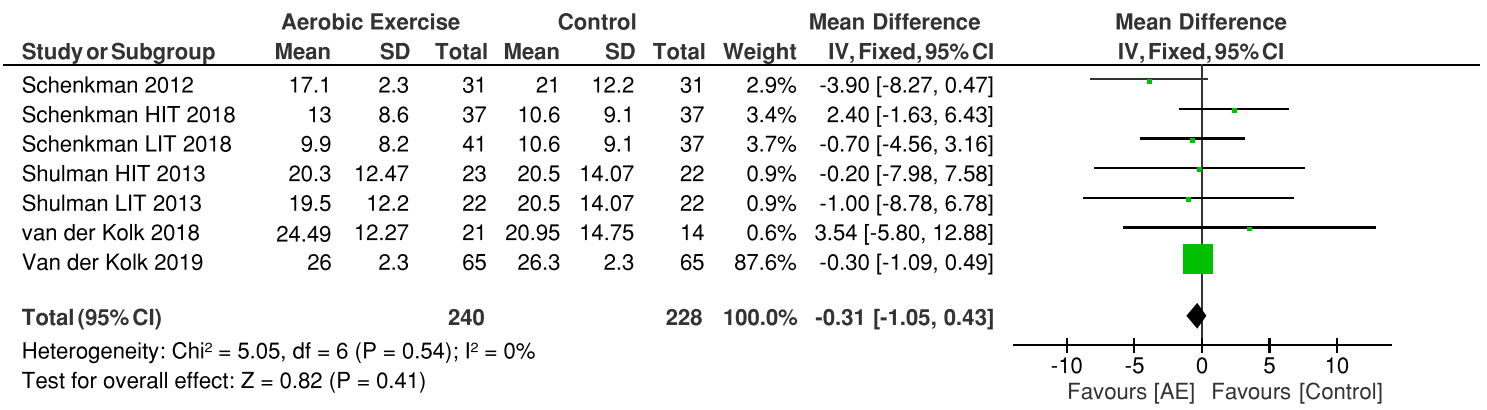

Fig. 5 Meta-analysis on the effect of aerobic exercise on health-related quality of life in PD comparing post-intervention PDQ-39 between the aerobic exercise group and the control group. $\mathrm{AE}=$ aerobic exercise group; HIT = high-intensity group; LIT = low-intensity group. 
patients: they offered support, tracked progression, and adjusted the training program via phone calls; compliance was excellent [4]. Interestingly, recent advances in mobile technology promise to offer a scalable alternative for intensively supervised interventions $[74,75]$. Apps could offer remote monitoring, give feedback, and allow for interaction with peers or others in the social environment. Few studies have thus far implemented such an approach in PD [4, 74], but mobile technologies certainly show promise for future exercise programs to boost compliance in daily life and may simultaneously be used to monitor their long-term effects in both research and clinical practice.

\section{Safety}

Although aerobic exercise studies in PD report low rates of exercise-related adverse events (five studies reported no adverse events [11, 28, 29, 32, 33], and one study reported that $2 \%$ of people [4] and another study $20.9 \%$ of people [23] in a high-intensity exercise group experienced an adverse event with a severity greater than mild, while this was the case for $8.9 \%$ of people in the moderate-intensity exercise group [23], there are some risks, especially in people with more advanced stages of PD. Obvious risks to consider are fall-related injuries and cardiovascular complications. Although the percentage of people with cardiac events that interfere with exercise was low in clinical trials, there is a theoretical concern that elderly and sedentary persons who start with a vigorous exercise program may experience cardiovascular complications. While the risk of cardiac adverse events is presumably low, the potential impact of a cardiac event is obviously substantial, including myocardial infarction or sudden death. Factors that increase fall-related injuries should be minimized by ascertaining that the exercise type matches the patient's physical capabilities. For example, people who experience freezing of gait should prefer exercising on a stationary home trainer over a treadmill. Also, exercising during a medication on state and at a time of the day when the patient feels subjectively best are general rules to reduce adverse events. We tell patients to consider taking an extra dose of medication prior to their exercise, in order to ascertain a good on state throughout the period of exercise. It is also important to inform patients about chronotropic incompetence, which is a common sign of autonomic dysfunction in PD [76] and which makes it more difficult for some patients to achieve the same exercise-related increase in heart rate [77]. This means that standardized heart rate zone estimates based on a person's age are often not reliable and that a (sub)maximal exercise test before commencing aerobic exercise is needed. A simple selfmanagement advice is to dose the exercise not based on heart rate, but rather to aim for an exercise that makes the patient pant, while still being able to maintain a conversation. Another strategy is to use the BORG scale of perceived effort
[78] although the patient needs to be educated in how to use this scale.

Another adverse effect to consider is that persons with PD have an increased risk of exercise-induced hypotension [79] and possibly also of post-exercise orthostatic hypotension [80]. A typical and worrisome manifestation is fainting after the patient has reached the top of a staircase, which can lead to injurious syncopal falls. Patients should be warned about this. Whether cardiovascular disease is indeed more prevalent in PD is still a matter of some debate, but like any person with known cardiovascular disease or with cardiovascular risk factors, it is prudent to consult a medical specialist before engaging in aerobic exercise. Also, some form of supervision is desirable to increase both adherence and safety. Especially heart rate monitoring allows for direct feedback to the patient and supervisor about each session's performance and about the overall training progress, bearing the limitations of a possible chronotropic incompetence in mind. It also reveals whether the training was performed at the right intensity and might suggest the presence of arrhythmias.

\section{Discussion}

We conclude that aerobic exercise offers generic health benefits, improves physical fitness, and offers symptomatic relief (reduced motor symptoms) in people with PD. This conclusion is supported by our meta-analyses on $\mathrm{VO}_{2}$ max and the (MDS-)UPDRS motor section in the off-medication state. More research is needed into the effect of aerobic exercise on non-motor symptoms and health-related quality of life.

What is the clinical significance of the observed increase in physical fitness and attenuation of motor symptoms for patients with PD? First, greater physical fitness may translate into cardiovascular health benefits. In the general population, higher doses of activity are associated with fewer cardiovascular complications [38] and we expect this to be the same for people with PD. A specific benefit would be a prophylactic effect on cerebrovascular disease, which commonly appears as a comorbid condition in PD; secondary prevention could help to arrest progression of symptoms such as gait disability or cognitive decline. This assumption should now be formally studied in people with PD. Second, it remains unknown whether the effects of medication and aerobic exercise are mediated through the same mechanism of enhanced dopaminergic signaling, or whether aerobic exercise has differential and synergistic effects. The attenuation of motor symptoms measured with the MDS-UPDRS motor section was predominantly observed during the medication off state in two trials $[4,23]$, so this may help patients with response fluctuations while they wait for their pharmacological treatment to kick in again. One of these two trials found no symptomatic relief in the on state [4]. Most other trials assessed motor symptoms 
only in the on state and showed conflicting results $[24,25,28$, $30,31]$. One possible explanation for this absent effect in the on state could be that the effect of medication is simply larger than the effect of aerobic exercise. Future studies should further address this issue, by including comprehensive assessments during both the medication off and on states as well as by using longer follow-up periods. Moreover, whether exercise can reverse or attenuate the underlying neurodegenerative processes in patients with PD is still unclear. Such effects are conceivable, realizing that exercise potentially creates optimal circumstances for neuroplasticity (i.e., increased angiogenesis, improved mitochondrial function, increased neurotrophic factors) $[6-8,81-85]$. Some earlier studies have certainly hinted at the possibility that (high-intensity) exercise could somehow alter the course of PD. For example, better physical fitness is associated with a larger brain volume, better white matter integrity, fewer white matter hyperintensities, and better connectivity patterns in healthy elderly persons [83-86]. Moreover, aerobic exercise afforded symptomatic improvements in neurotoxic animal models of $\mathrm{PD}$, which upon postmortem brain examination proved to be accompanied by adaptive neuroplasticity that promoted an increase in dopaminergic neurotransmission [6-8, 81-85]. In persons with PD, preliminary data from small aerobic exercise studies provided similar results, suggesting enhanced dopaminergic signaling $[10,86]$ as well as increased brain activity in the basal ganglia and motor cortex [87, 88]. Disentangling symptomatic from possible disease-modifying effects in humans remains incredibly difficult, but future exercise trials could consider including a washout period or a delayed start design, as well as inclusion of surrogate imaging outcomes (dopamine scans and/or structural/functional MRI). This exciting area definitely warrants further research attention.

There is insufficient evidence for a beneficial effect of aerobic exercise on gait, balance, falls, and functional mobility. It is possible that aerobic exercise alone, given its relatively weak effects compared to medication that we alluded to earlier [4], is unable to overcome such rather gross deficits in axial mobility. One option is therefore to consider combining aerobic exercise with task-specific (gait) training if patients manifest impairments in gait, balance, or functional mobility.

The effects of aerobic exercise on non-motor symptoms and health-related quality of life in PD were thus far unconvincing. However, several important limitations in the available evidence must be considered here. First, non-motor symptoms and health-related quality of life were never included as primary endpoint. Five studies included improvement of non-motor symptoms as the main aim $[25,26,28,36$, $37]$, but none specified one primary outcome or reported a sample size calculation (except [36]). Therefore, these studies may have been underpowered to detect improvements in nonmotor symptoms or health-related quality of life. Second, study candidates were not selected based on the presence of non-motor symptoms or poor health-related quality of life. Consequently, participants manifested few depressive symptoms and cognitive deficits, while baseline health-related quality of life was high, which limited the likelihood of finding an effect on these symptoms. This is supported by a study showing that aerobic exercise did improve cognitive functioning (as measured with the Trail Making Test B) in people with PD who had cognitive impairment, as compared with usual care controls who also had cognitive impairment [37]. Third, trials were relatively short (4-24 weeks), but more prolonged interventions may be needed to find an effect. Fourth, virtually no studies reported the effect of aerobic exercise on sleep $[4,25,33]$, fatigue $[4,28,31]$, and constipation [4], even though all these domains are theoretically attractive targets for improvement with exercise. This area therefore warrants further research. Future trials should specifically target people with PD who experience depression or anxiety, apathy, cognitive problems, sleep problems, fatigue, or reduced health-related quality of life to elucidate the effect of aerobic exercise on these non-motor symptoms and on health-related quality of life.

While evidence for a beneficial effect of aerobic exercise is emerging, much work remains to be done. First, large trials with longer follow-up periods are needed to determine the long-term effects and compliance. Second, future studies must determine the optimal and minimal dose (frequency, duration, and intensity) of exercise. So far, only one highquality study investigated the effect of different doses of aerobic exercise in PD [23]. To optimize the aerobic exercise training sessions, we need more studies investigating the safety and effectiveness of different doses of exercise. Third, we require trials that investigate the effect of different modes of aerobic exercise. We here reviewed studies that investigated the effect of different modes of aerobic exercise-mainly treadmill training and cycle ergometry - but none have directly compared both types. Fourth, research into the potential disease-modifying effect of exercise should be high on the research agenda. Gaining knowledge into the underlying mechanism of disease modification would also be a major motivator to get people engaged in exercise. Fifth, we need a more objective measure of PD motor symptoms. The (MDS-)UPDRS motor section is the gold standard, but the clinical relevance can be questioned as it only provides a snapshot of in-clinic performance that may not reflect actual daily life performance [89]. Wearable sensors allow for continuous and unobtrusive measurement in daily life. However, digital biomarkers derived from such sensors remain to be developed and have yet to prove their value as outcomes in clinical trials. Finally, to promote aerobic exercise even in remote, loosely populated or underdeveloped areas of the world, we need scalable approaches. Wearable sensors and remote monitoring through apps could be useful here, but their merits also remain to be proven in future trials [89]. 
Acknowledgments The Center of Expertise for Parkinson \& Movement Disorders was supported by a center of excellence grant of the Parkinson's Foundation. N.M. de Vries and S. Schootemeijer were financially supported by ZonMw (The Netherlands Organisation for Health Research and Development; grant numbers 91619142 and 546003007).

Required Author Forms Disclosure forms provided by the authors are available with the online version of this article.

\section{Compliance with Ethical Standards}

Conflict of Interest The authors have no conflict of interest to report.

Open Access This article is licensed under a Creative Commons Attribution 4.0 International License, which permits use, sharing, adaptation, distribution and reproduction in any medium or format, as long as you give appropriate credit to the original author(s) and the source, provide a link to the Creative Commons licence, and indicate if changes were made. The images or other third party material in this article are included in the article's Creative Commons licence, unless indicated otherwise in a credit line to the material. If material is not included in the article's Creative Commons licence and your intended use is not permitted by statutory regulation or exceeds the permitted use, you will need to obtain permission directly from the copyright holder. To view a copy of this licence, visit http://creativecommons.org/licenses/by/4.0/.

\section{References}

1. Dorsey ER, Bloem BR. The Parkinson Pandemic-A Call to Action. JAMA Neurol 2018;75(1):9-10.

2. Ahlskog JE, Muenter MD. Frequency of levodopa-related dyskinesias and motor fluctuations as estimated from the cumulative literature. Mov Disord 2001;16(3):448-458.

3. Bronstein JM, Tagliati M, Alterman RL, et al. Deep brain stimulation for Parkinson disease: an expert consensus and review of key issues. Arch Neurol 2011;68(2):165.

4. van der Kolk NM, de Vries NM, Kessels RPC, et al. Effectiveness of home-based and remotely supervised aerobic exercise in Parkinson's disease: a double-blind, randomised controlled trial. Lancet Neurol 2019;18(11):998-1008.

5. Piercy KL, Troiano RP, Ballard RM, et al. The Physical Activity Guidelines for Americans. JAMA. 2018;320(19):2020-2028.

6. Dutra MF, Jaeger M, Ilha J, Kalil-Gaspar PI, Marcuzzo S, Achaval M. Exercise improves motor deficits and alters striatal GFAP expression in a 6-OHDA-induced rat model of Parkinson's disease. Neurol Sci 2012;33(5):1137-44

7. Petzinger GM, Walsh JP, Akopian G, et al. Effects of treadmill exercise on dopaminergic transmission in the 1-methyl-4-phenyl1,2,3,6-tetrahydropyridine-lesioned mouse model of basal ganglia injury. J Neurosci 2007;27(20):5291-5300.

8. Steiner B, Winter C, Hosman K, et al. Enriched environment induces cellular plasticity in the adult substantia nigra and improves motor behavior function in the 6-OHDA rat model of Parkinson's disease. Exp Neurol 2006;199(2):291-300.

9. Churchill MJ, Pflibsen L, Sconce MD, Moore C, Kim K, Meshul CK. Exercise in an animal model of Parkinson's disease: Motor recovery but not restoration of the nigrostriatal pathway. Neuroscience. 2017:359:224-47.
10. Fisher BE, Li Q, Nacca A, et al. Treadmill exercise elevates striatal dopamine D2 receptor binding potential in patients with early Parkinson's disease. Neuroreport. 2013;24(10):509-514.

11. Fisher BE, $\mathrm{Wu} \mathrm{AD}$, Salem GJ, et al. The effect of exercise training in improving motor performance and corticomotor excitability in people with early Parkinson's disease. Arch Phys Med Rehabil 2008;89(7):1221-1229.

12. Ascherio A, Schwarzschild MA. The epidemiology of Parkinson's disease: risk factors and prevention. Lancet Neurol 2016;15(12): $1257-1272$.

13. Yang F, Trolle Lagerros Y, Bellocco R, et al. Physical activity and risk of Parkinson's disease in the Swedish National March Cohort. Brain. 2015;138(Pt 2):269-275.

14. Chen H, Zhang SM, Schwarzschild MA, Hernán MA, Ascherio A. Physical activity and the risk of Parkinson disease. Neurology. 2005;64(4):664-669.

15. Saaksjarvi K, Knekt P, Mannisto S, et al. Reduced risk of Parkinson's disease associated with lower body mass index and heavy leisure-time physical activity. Eur J Epidemiol 2014;29(4): 285-292.

16. Fang X, Han D, Cheng Q, et al. Association of Levels of Physical Activity With Risk of Parkinson Disease: A Systematic Review and Meta-analysis. JAMA Netw Open 2018;1(5):e182421.

17. Lamotte G, Rafferty MR, Prodoehl J, et al. Effects of endurance exercise training on the motor and non-motor features of Parkinson's disease: a review. J Parkinsons Dis 2015;5(1):21-41.

18. Norton K, Norton L, Sadgrove D. Position statement on physical activity and exercise intensity terminology. J Sci Med Sport 2010;13(5):496-502.

19. Ridgel AL, Vitek JL, Alberts JL. Forced, not voluntary, exercise improves motor function in Parkinson's disease patients. Neurorehabil Neural Repair 2009;23(6):600-608.

20. Higgins JPT, Thompson SG, Deeks JJ, Altman DG. Measuring inconsistency in meta-analyses. BMJ 2003;327(7414):7557-60.

21. Begg CB. Publication bias. The handbook of research synthesis. New York: Russell Sage Foundation; 1994. p. 399-409.

22. Sterne JAC, Savovic J, Page MJ, et al. RoB 2: a revised tool for assessing risk of bias in randomised trials. BMJ. 2019;366:14898.

23. Schenkman M, Moore CG, Kohrt WM, et al. Effect of HighIntensity Treadmill Exercise on Motor Symptoms in Patients With De Novo Parkinson Disease: A Phase 2 Randomized Clinical Trial. JAMA Neurol 2018;75(2):219-226.

24. Schenkman M, Hall DA, Baron AE, Schwartz RS, Mettler P, Kohrt WM. Exercise for people in early- or mid-stage Parkinson disease: a 16-month randomized controlled trial. Phys Ther 2012;92(11): 1395-1410.

25. Altmann LJ, Stegemoller E, Hazamy AA, et al. Aerobic Exercise Improves Mood, Cognition, and Language Function in Parkinson's Disease: Results of a Controlled Study. J Int Neuropsychol Soc 2016;22(9):878-889.

26. Bridgewater KJ, Sharpe MH. Aerobic Exercise and Early Parkinson's Disease. J Neurol Rehabil 1996;10(4):233-241.

27. Carvalho A, Barbirato D, Araujo N, et al. Comparison of strength training, aerobic training, and additional physical therapy as supplementary treatments for Parkinson's disease: pilot study. Clin Interv Aging 2015;10:183-191.

28. Cugusi L, Solla P, Serpe R, et al. Effects of a Nordic Walking program on motor and non-motor symptoms, functional performance and body composition in patients with Parkinson's disease. NeuroRehabilitation. 2015;37(2):245-254

29. Sacheli MA, Neva JL, Lakhani B, et al. Exercise increases caudate dopamine release and ventral striatal activation in Parkinson's disease. Mov Disord 2019;34(12):1891-1900.

30. Sage MD, Almeida QJ. Symptom and gait changes after sensory attention focused exercise vs aerobic training in Parkinson's disease. Mov Disord 2009;24(8):1132-1138. 
31. Shulman LM, Katzel LI, Ivey FM, et al. Randomized clinical trial of 3 types of physical exercise for patients with Parkinson disease. JAMA Neurol 2013;70(2):183-190.

32. Tollar J, Nagy F, Hortobagyi T. Vastly Different Exercise Programs Similarly Improve Parkinsonian Symptoms: A Randomized Clinical Trial. Gerontology. 2019;65(2):120-127.

33. van der Kolk NM, de Vries NM, Penko AL, et al. A remotely supervised home-based aerobic exercise programme is feasible for patients with Parkinson's disease: results of a small randomised feasibility trial. J Neurol Neurosurg Psychiatry 2018;89(9):10031005.

34. Bergen JL, Toole T, Elliott RG, 3rd, Wallace B, Robinson K, Maitland CG. Aerobic exercise intervention improves aerobic capacity and movement initiation in Parkinson's disease patients. NeuroRehabilitation. 2002;17(2):161-168.

35. Kurtais Y, Kutlay S, Tur BS, Gok H, Akbostanci C. Does treadmill training improve lower-extremity tasks in Parkinson disease? A randomized controlled trial. Clin J Sport Med 2008;18(3):289-291.

36. Marusiak J, Fisher BE, Jaskolska A, et al. Eight Weeks of Aerobic Interval Training Improves Psychomotor Function in Patients with Parkinson's Disease-Randomized Controlled Trial. Int J Environ Res Public Health. 2019;16(5):880

37. Silveira CRA, Roy EA, Intzandt BN, Almeida QJ. Aerobic exercise is more effective than goal-based exercise for the treatment of cognition in Parkinson's disease. Brain Cogn 2018;122:1-8.

38. Eijsvogels TM, Molossi S, Lee DC, Emery MS, Thompson PD. Exercise at the Extremes: The Amount of Exercise to Reduce Cardiovascular Events. J Am Coll Cardiol 2016;67(3):316-329.

39. Saklayen MG. The Global Epidemic of the Metabolic Syndrome. Curr Hypertens Rep 2018;20(2):12.

40. Leehey M, Luo S, Sharma S, et al. Association of metabolic syndrome and change in Unified Parkinson's Disease Rating Scale scores. Neurology. 2017;89(17):1789-1794.

41. Peng Z, Dong S, Tao Y, et al. Metabolic syndrome contributes to cognitive impairment in patients with Parkinson's disease. Parkinsonism Relat Disord 2018;55:68-74.

42. Bohnen NI, Albin RL. White matter lesions in Parkinson disease. Nat Rev Neurol 2011;7(4):229-236.

43. Van Der Holst HM, van Uden IW, Tuladhar AM, et al. Cerebral small vessel disease and incident parkinsonism: the RUN DMC study. Neurology. 2015;85(18):1569-1577.

44. Nanhoe-Mahabier W, de Laat KF, Visser JE, Zijlmans J, de Leeuw FE, Bloem BR. Parkinson disease and comorbid cerebrovascular disease. Nat Rev Neurol 2009;5(10):533-541.

45. van den Bos F, Speelman AD, Samson M, Munneke M, Bloem BR, Verhaar HJ. Parkinson's disease and osteoporosis. Age Ageing 2013;42(2):156-162.

46. Invernizzi M, Carda S, Viscontini GS, Cisari C. Osteoporosis in Parkinson's disease. Parkinsonism Relat Disord 2009;15(5):339346.

47. Bezza A, Ouzzif Z, Naji H, et al. Prevalence and risk factors of osteoporosis in patients with Parkinson's disease. Rheumatol Int 2008;28(12):1205-1209.

48. Metta V, Sanchez TC, Padmakumar C. Osteoporosis: A Hidden Nonmotor Face of Parkinson's Disease. Int Rev Neurobiol 2017;134:877-890.

49. Logroscino G, Sesso HD, Paffenbarger RS, Jr., Lee IM. Physical activity and risk of Parkinson's disease: a prospective cohort study. J Neurol Neurosurg Psychiatry 2006;77(12):1318-1322.

50. Thacker EL, Chen H, Patel AV, et al. Recreational physical activity and risk of Parkinson's disease. Mov Disord 2008;23(1):69-74.

51. $\mathrm{Xu} \mathrm{Q}$, Park $\mathrm{Y}$, Huang $\mathrm{X}$, et al. Physical activities and future risk of Parkinson disease. Neurology. 2010;75(4):341-348.

52. Olsson TT, Svensson M, Hallmarker U, James S, Deierborg T. Delayed Clinical Manifestation of Parkinson's Disease Among
Physically Active: Do Participants in a Long-Distance Ski Race Have a Motor Reserve? J Parkinsons Dis 2020;10(1):267-274.

53. Sunwoo MK, Lee JE, Hong JY, et al. Premorbid exercise engagement and motor reserve in Parkinson's disease. Parkinsonism Relat Disord 2017;34:49-53.

54. Hughes KC, Gao X, Molsberry S, Valeri L, Schwarzschild MA, Ascherio A. Physical activity and prodromal features of Parkinson disease. Neurology. 2019;93(23):e2157-e2169.

55. Ascherio A, Schwarzschild MA. Lifestyle and Parkinson's disease progression. Mov Disord 2019;34(1):7-8.

56. Rafferty MR, Schmidt PN, Luo ST, et al. Regular Exercise, Quality of Life, and Mobility in Parkinson's Disease: A Longitudinal Analysis of National Parkinson Foundation Quality Improvement Initiative Data. J Parkinsons Dis 2017;7(1):193-202.

57. Afshari M, Yang A, Bega D. Motivators and Barriers to Exercise in Parkinson's Disease. J Parkinsons Dis 2017;7(4):703-711.

58. Ellis T, Boudreau JK, DeAngelis TR, et al. Barriers to exercise in people with Parkinson disease. Phys Ther 2013;93(5):628-636.

59. Hunter H, Lovegrove C, Haas B, Freeman J, Gunn H. Experiences of people with Parkinson's disease and their views on physical activity interventions: a qualitative systematic review. JBI Database System Rev Implement Rep 2019;17(4):548-613.

60. Mantri S, Wood S, Duda JE, Morley JF. Understanding physical activity in Veterans with Parkinson disease: A mixed-methods approach. Parkinsonism Relat Disord 2019;61:156-160.

61. Zaman A, Ellingson L, Sunken A, Gibson E, Stegemoller EL. Determinants of exercise behaviour in persons with Parkinson's disease. Disabil Rehabil 2019;1-7.

62. Ellis T, Rochester L. Mobilizing Parkinson's Disease: The Future of Exercise. J Parkinsons Dis 2018;8(s1):S95-S100.

63. O'Brien C, Clemson L, Canning CG. Multiple factors, including non-motor impairments, influence decision making with regard to exercise participation in Parkinson's disease: a qualitative enquiry. Disabil Rehabil 2015;38(5):472-481

64. Speelman AD, van Nimwegen M, Bloem BR, Munneke M. Evaluation of implementation of the ParkFit program: A multifaceted intervention aimed to promote physical activity in patients with Parkinson's disease. Physiotherapy. 2014;100(2):134-341.

65. Ene H, McRae C, Schenkman M. Attitudes toward exercise following participation in an exercise intervention study. J Neurol Phys Ther 2011;35(1):34-40.

66. Urell C, Zetterberg L, Hellstrom K, Anens E. Factors explaining physical activity level in Parkinson s disease: A gender focus. Physiother Theory Pract 2019;1-10.

67. Sheehy TL, McDonough MH, Zauber SE. Social Comparisons, Social Support, and Self-Perceptions in Group Exercise for People With Parkinson's Disease. J Appl Sport Psychol 2016;29(3):285-303.

68. Stevens A, Stanton R, Rebar AL. Helping People With Parkinson Disease Build Exercise Self-Efficacy. Phys Ther 2020;100(2):205208.

69. Mak MK, Wong-Yu IS, Shen X, Chung CL. Long-term effects of exercise and physical therapy in people with Parkinson disease. Nat Rev Neurol 2017;13(11):689-703.

70. Ellis T, Cavanaugh JT, Earhart GM, et al. Factors Associated with Exercise Behavior in Persons with Parkinson Disease. Phys Ther 2011;91:1838-1848.

71. Lamont RM, Daniel HL, Payne CL, Brauer SG. Accuracy of wearable physical activity trackers in people with Parkinson's disease. Gait Posture 2018;63:104-108.

72. Ashburn A, Pickering R, McIntosh E, et al. Exercise- and strategybased physiotherapy-delivered intervention for preventing repeat falls in people with Parkinson's: the PDSAFE RCT. Health Technol Assess 2019;23(36):1-150. 
73. Lamont RM, Morris ME, Woollacott MH, Brauer SG. Community walking in people with Parkinson's disease. Parkinsons Dis 2012;2012:856237.

74. Ellis TD, Cavanaugh JT, DeAngelis T, et al. Comparative Effectiveness of mHealth-Supported Exercise Compared With Exercise Alone for People With Parkinson Disease: Randomized Controlled Pilot Study. Phys Ther 2019;99(2):203-216.

75. Romeo A, Edney S, Plotnikoff R, et al. Can Smartphone Apps Increase Physical Activity? Systematic Review and MetaAnalysis. J Med Internet Res 2019;21(3):e12053.

76. Merola A, Romagnolo A, Rosso M, et al. Autonomic dysfunction in Parkinson's disease: A prospective cohort study. Mov Disord 2018;33(3):391-397.

77. Speelman AD, Groothuis JT, van Nimwegen M, et al. Cardiovascular responses during a submaximal exercise test in patients with Parkinson's disease. J Parkinsons Dis 2012;2(3): 241-247.

78. Borg G. Borg's perceived exertion and pain scales. Champaign, IL, US: Human Kinetics; 1998. viii, 104-viii, p.

79. Low DA, Vichayanrat E, Iodice V, Mathias CJ. Exercise hemodynamics in Parkinson's disease and autonomic dysfunction. Parkinsonism Relat Disord 2014;20(5):549-553.

80. Smith GD, Mathias CJ. Postural hypotension enhanced by exercise in patients with chronic autonomic failure. QJM. 1995;88(4):251256.

81. Caudle WM, Tillerson JL, Reveron ME, Miller GW. Usedependent behavioral and neurochemical asymmetry in MPTP mice. Neurosci Lett 2007;418(3):213-216.

82. Faherty CJ, Raviie Shepherd K, Herasimtschuk A, Smeyne RJ. Environmental enrichment in adulthood eliminates neuronal death in experimental Parkinsonism. Brain Res Mol Brain Res 2005;134(1):170-179.

83. VanLeeuwen JE, Petzinger GM, Walsh JP, Akopian GK, Vuckovic M, Jakowec MW. Altered AMPA receptor expression with treadmill exercise in the 1-methyl-4-phenyl-1,2,3,6-tetrahydropyridinelesioned mouse model of basal ganglia injury. J Neurosci Res 2010;88(3):650-668.

84. Vuckovic MG, Li Q, Fisher B, et al. Exercise elevates dopamine D2 receptor in a mouse model of Parkinson's disease: in vivo imaging with [(1)(8)F]fallypride. Mov Disord 2010;25(16):2777-2784.

85. Al-Jarrah M, Jamous M, Al Zailaey K, Bweir SO. Endurance exercise training promotes angiogenesis in the brain of chronic/ progressive mouse model of Parkinson's Disease. NeuroRehabilitation. 2010;26(4):369-373.

86. Sacheli MA, Murray DK, Vafai N, et al. Habitual exercisers versus sedentary subjects with Parkinson's Disease: Multimodal PET and fMRI study. Mov Disord 2018;33(12):1945-1950.

87. Duchesne C, Gheysen F, Bore A, et al. Influence of aerobic exercise training on the neural correlates of motor learning in Parkinson's disease individuals. Neuroimage Clin 2016;12:559-569.

88. Shah C, Beall EB, Frankemolle AM, et al. Exercise Therapy for Parkinson's Disease: Pedaling Rate Is Related to Changes in Motor Connectivity. Brain Connect 2016;6(1):25-36.

89. Warmerdam E, Hausdorff JM, Atrsaei A, et al. Long-term unsupervised mobility assessment in movement disorders. Lancet Neurol 2020;19(5):462-470.

Publisher's Note Springer Nature remains neutral with regard to jurisdictional claims in published maps and institutional affiliations. 